\title{
Relationship between Chinese medicine dietary patterns and the incidence of breast cancer in Chinese women in Hong Kong: a retrospective cross-sectional survey
}

Xiao Zheng ${ }^{1,2}$, Jianping Chen ${ }^{1,5^{*}}$, Ting Xie ${ }^{2}$, Zhiyu Xia ${ }^{3}$, Wings Tjing Yung Loo' ${ }^{1}$ Lixing Lao ${ }^{1}$, JieShu You', Jie Yang ${ }^{5}$, Kamchuen Tsui ${ }^{4}$, Feizhi Mo ${ }^{1}$ and Fei Gao ${ }^{1}$

\begin{abstract}
Background: This retrospective cross-sectional study aimed to investigate the relationship between Chinese medicine (CM) dietary patterns (hot, neutral, and cold) and the incidence of breast cancer among Chinese women in Hong Kong.

Methods: Breast cancer cases $(n=202)$ and healthy controls $(n=202)$ were matched according to demographics. Chinese women residing in Hong Kong for the past 7 years were recruited by media advertisements (e.g., via newspapers, radio, and posters). The control participants were recruited by convenience sampling from health workshops held in clinics and communities of 15 districts of Hong Kong. After completing test-retest reliability, all participants were asked to complete diet pattern questionnaires about their food preferences and dietary patterns. The Student's unpaired $t$ test, Chi square test, and logistic regression were conducted using SPSS software.

Results: Three major CM dietary patterns were identified: hot, neutral, and cold. The participants with breast cancer exhibited a stronger preference for hot food than the control group (Chi square test, $P<0.001$ ). A higher frequency of breast cancer was associated with a higher frequency of dining out for breakfast (4-5 times per week, Chi square test, $P=0.015 ; 6-7$ times per week, Chi square test, $P<0.001$ ) and lunch ( $4-5$ times per week, Chi square test, $P<0.001$; $6-7$ times per week, Chi square test, $P=0.006)$. The participants with no history of breast cancer consumed CM supplements and Guangdong soups ( $1-2$ times per week, Chi square test, $P=0.05 ;>3$ times per week, Chi square test, $P<0.001)$ more frequently than those with breast cancer.
\end{abstract}

Conclusions: Non-breast cancer participants adopted a neutral (healthy and balanced) dietary pattern, and consumed CM supplements and Guangdong soups more frequently.

\section{Background}

Breast cancer is the most common and one of the most fatal cancers in women worldwide. The number of breast cancer cases among women in Hong Kong increased from 2273 in 2004 to more than 2870 in 2009, and average incidence rate per 100,000 women between 2004 and

\footnotetext{
*Correspondence: jpjpchen@yahoo.com; abchen@hku.hk

${ }^{\dagger}$ Xiao Zheng and Jianping Chen are co-first authors

${ }^{1}$ School of Chinese Medicine, The University of Hong Kong, Hong Kong, China

Full list of author information is available at the end of the article
}

2009 was 386.7 [1]. In 2008, the lifetime risk of breast cancer was 1 in 20 women [2]. Breast cancer incidence was generally lower in Asia than in Europe in 2000 [3]; a key reason for this was the difference in dietary patterns between the East and the West [4]. The identification of reasonable dietary prevention methods and the development of corresponding inhibitors may be beneficial for breast cancer prevention [5]. However, little research has been conducted in this area.

The dietary patterns of women in Hong Kong have been changing with western lifestyle in recent years $[6,7]$. The 
intake of foods with certain properties (e.g., hot or cold features) may increase the risk of related diseases, such as cancers [8]. Although diet has been proposed as a possible cause of breast cancer [9-11], the incidence of breast cancer has not previously been related to Chinese medicine (CM)-defined dietary patterns.

The properties of CM material medica (cold, cool, hot, and warm) can be used to classify diets as hot, neutral, or cold; these types of food have different functions in the body. Under this classification, beef, shrimp, chicken, and even ice cream are hot foods (Additional file 1).

This study aimed to investigate the relationship between CM dietary patterns and the incidence of breast cancer among women in Hong Kong.

\section{Methods}

\section{Study design and participants}

A retrospective cross-sectional survey was conducted. Data were collected using the Food Frequency Questionnaires (FFQ) (Additional file 2) [12]. The selection of study participants was based on geographic and sociodemographic variables described previously $[6,8,9]$. The Minimum Standards of Reporting Checklist (Additional file 3) contain details of the experimental design, and statistics, and resources used in this study.

This study included female participants with breast cancer (cases), who were compared with healthy women (controls). The cases were recruited by advertisements in the local media, such as in newspapers, in booklets issued by the University of Hong Kong, on posters, and on the radio. Convenience sampling was used to recruit the control group from health workshops in clinics and communities in 15 Hong Kong districts. All participants had to fulfill the following conditions: (1) female, (2) Chinese women residing in Hong Kong, (3) 28-60 years of age, (4) living in Hong Kong for the past 7 years, (5) able to understand the questionnaire items in Chinese, and (6) provision of an informed consent. Controls were required to fulfill the following conditions: (1) no breast cancer or other gynecologic diseases; (2) no metabolic, nutritional, or other severe diseases; and (3) no serious disease after doctor consultation in the past 3 months. Case inclusion criteria were: (1) new primary breast cancer patients, and (2) a diagnosis of primary breast cancer through biopsy and/or discharge report. Participants were excluded from the study if they had (1) other prior cancer diagnosis, (2) treatments with psychotropic drugs, (3) any medical conditions that limited their physical activity, and (4) not completed the questionnaire.

\section{Sample size}

We conducted a pilot study with 37 participants whose total diet comprised mainly hot foods and an expected odds ratio (OR) of 2.32 according to the formula of OR was found. For the pilot study, we invited 37 people from the oncology outpatient clinic and the general outpatient clinic in the School of Chinese Medicine, University of Hong Kong, to complete the questionnaire: 20 were breast cancer cases and 17 were not. After the pilot study, we analyzed the responses and calculated the consumption of 12 kinds of hot foods including beef, mutton, shrimp, soft drinks, and milk. Of the breast cancer cases, $45.8 \%$ consumed hot foods regularly, whereas only $26.7 \%$ of the controls did so. We chose $10 \%$ as the estimated prevalence of hot food consumption for control group and calculated the estimated prevalence of hot food consumption for breast cancer group as 0.2 the expected OR of 2.32 to estimate the sample size of our research. The estimated sample size was calculated as about 207 participants in each group. Taking into account the nullified questionnaires, 202 participants were eventually included out of the total 207 participants recruited in each group.

\section{Questionnaire}

A self-administered FFQ was used to collect the dietary intake information. This questionnaire was adapted from the Fred Hutchinson Cancer Research Center Food Questionnaire, which is based on Western dietary patterns $[13,14]$. Two CM experts and a group of laypeople approved the revised questionnaire as suitable to assess the average dietary patterns of Chinese women in Hong Kong. The FFQ consisted of 74 food items (Additional file 4). A commonly used portion size was specified for each food based on the conventional food intake and dietary patterns of Hong Kong women.

The questionnaire consists of two sections. The first section assessed sociodemographic information, including age, working status, education level, weight, height, and marital status. The second section contained the CM FFQ items, which measured consumption of very common foods. The FFQ consists of 74 items scored on a 5-point scale, ranging from 1 (not consumed at all) to 5 (consumed very often). It comprises nine subscales of food consumption frequency for CM-defined foods, and a general preference score for three kinds of food (cold, neutral, and hot foods, classified according to CM). A total score for each subscale was obtained by summing the relevant item scores. Higher scores indicated higher consumption frequencies [15]. We modified the semantic expression of several items to adapt those items to the dietary patterns and habits of the women in Hong Kong. Our pilot study of 37 participants demonstrated that the version could be read and understood by local Chinese women.

The questionnaire design was single-blind to participants to ensure the validity of participants' stated consumption of their preferred and most frequently eaten 
foods. Different kinds of food were included and randomized to ensure the aim of the survey was not revealed to the cases or controls. Completed questionnaires were collected individually to ensure the responses were not misinterpreted owing to communication gaps between the researchers and the participants.

\section{Study procedures}

Test-retest reliability was examined using pre-survey responses. Two weeks after completing the first questionnaire, we asked the same participants to complete the same questionnaire to confirm the instrument's validity and test-retest reliability. Analysis of homogeneity in this pilot study result was used to test the consistency of the 37 completed questionnaires (20 cases and 17 controls). The results produced a $P$ value of 0.915 , which indicated that the questionnaire responses were statistically the same for the two tests. Each participant was asked to appraise each item for its relevance in evaluating the content, and to suggest any items that should be added to improve the questionnaire's validity. All items had a content validity index of clarity greater than $80 \%$.

Every potential participant was interviewed by a trained research doctor at the clinic center of the School of Chinese Medicine, University of Hong Kong. Participants completed a self-administered questionnaire and had a face-to-face consultation for the same research doctor to check the questionnaire.

Three important steps were taken throughout the process to ensure the quality of investigation. First, to ensure consistency of the survey across sites and over time, the same interviewer used the same procedures and standards to investigate all women. Second, each questionnaire was checked by the same researcher carefully and those questionnaires with missing data on foods or dietary patterns were excluded. Third, to prevent input error, we used a double data entry method by different researchers to enter all data into SPSS.

\section{Measurement}

According to $\mathrm{CM}$ theory, different foods possess different cold, neutral, and hot properties and have different nutritional constituents, each with their own cold, neutral, and hot properties. These food properties are based on the functions the foods perform after consumption. All vegetables, meat, fruits, and grains are classified according to one of these three properties, as summarized in Additional file 1. Participants' final overall food preference score of $h o t$, neutral, or cold was determined by the average score for all the foods listed. For example, if the overall FFQ score for neutral food was the highest, then the overall preference score for food was considered neutral. The hot and cold preference scores were calculated in the same manner.
Additionally, participants were asked to indicate the frequency of (1) eating out for breakfast, lunch, and dinner (0-1 time/week; 2-3 times/week; 4-5 times/week, and 6-7 times/week); (2) CM intake, CM supplement intake, and Chinese soup consumption (seldom, sometimes, often); and (3) Chinese-style food (i.e., Guangdong $\mathrm{dim}$ sum/tea drinking) and Western-style food consumption (i.e., fast food) (<once/week; 1-2 times/week; >3 times/week). Demographic information such as age, marital status, current height and weight (to obtain body mass index), and education level was also collected.

\section{Data analysis}

The statistics program SPSS version 16.0 (SPSS Inc., Chicago, IL, USA) was used for data analysis. Descriptive statistics were calculated; continuous variables were presented as mean \pm standard deviation, and categorical variables were presented as frequencies. The mean difference between continuous variables was tested using Student's $t$ test for independent samples. The association between categorical variables was tested using the Chi square test. A binary logistic regression model was used to evaluate the association of different food preferences with the likelihood of having breast cancer. Results were presented as ORs and corresponding 95\% confidence intervals, with age as a variable in the logistic regression. All $P$ values were based on twotailed tests, with $P<0.05$ considered statistically significant.

Regarding the validity and reliability assessment of the questionnaire, we examined test-retest reliability by inviting participants in the pilot study to complete the questionnaire a second time. We compared both test results using ANOVA, which produced a value of 0.915 , showing high test-retest reliability. All the pilot study participants were interviewed about the interpretability of the questionnaire, and the interpretability was reported to be good.

\section{Results \\ Sociodemographic characteristics of participants}

The case and control groups were similar in terms of body mass index, occupation, education, marital status, age at first menstrual cycle, and family history of cancer (Table 1).

When age was treated as a continuous variable, there was a significant difference between the case and control groups $(P<0.001)$ : participants in the control group were 2.4 years younger. However, when age was categorized according to five different age groups, there was no significant difference between the case and control groups $(P=0.061)$. Because there was a marginally significant difference in age between the two groups, we considered age a confounder in multivariate analysis of variance (MANOVA). 
Table 1 Socio-demographic characteristics of participants $(N=404, \%)$

\begin{tabular}{|c|c|c|c|}
\hline Socio-demographic characteristics & Controls $(n=202)$ & Cases $(n=202)$ & $P$ values \\
\hline Age & & & 0.061 \\
\hline Mean \pm SD & $45.35 \pm 6.765$ & $47.75 \pm 6.17$ & \\
\hline$\leq 34$ & $14(6.9)$ & $7(3.5)$ & \\
\hline $35-41$ & $39(19.3)$ & $25(12.4)$ & \\
\hline $42-48$ & $76(37.6)$ & $72(36.6)$ & \\
\hline $49-55$ & $62(30.7)$ & $78(39.0)$ & \\
\hline$\geq 56$ & $11(5.4)$ & $18(9.0)$ & \\
\hline BMl & & & 0.132 \\
\hline Underweight (<18.5) & $17(8.5)$ & $19(9.5)$ & \\
\hline Normal weight (18.5-24.9) & $143(71.1)$ & $156(78.4)$ & \\
\hline Overweight (25-29.9) & $33(16.4)$ & $21(10.6)$ & \\
\hline Obesity $(\geq 30)$ & $8(4)$ & $3(1.5)$ & \\
\hline Occupational type & & & 0.589 \\
\hline Full-time & $119(59.5)$ & $113(55.9)$ & \\
\hline Part-time & $14(7.0)$ & $22(10.9)$ & \\
\hline Housewife & $66(33.0)$ & $66(32.7)$ & \\
\hline Others & $1(0.5)$ & $1(0.5)$ & \\
\hline Educational level & & & 0.063 \\
\hline Primary & $21(10.4)$ & $24(11.9)$ & \\
\hline Secondary & $92(45.5)$ & $115(56.9)$ & \\
\hline Tertiary or above & $87(43.1)$ & $62(30.7)$ & \\
\hline Others & $2(1.0)$ & $1(0.5)$ & \\
\hline Marital status & & & 0.389 \\
\hline Single & $33(16.3)$ & $25(12.4)$ & \\
\hline Married or cohabited & $155(76.7)$ & $158(78.2)$ & \\
\hline Divorced & $14(6.9)$ & $19(9.4)$ & \\
\hline Family tumor history & & & 0.318 \\
\hline Yes & $87(43.1)$ & $97(48.0)$ & \\
\hline No & $115(56.9)$ & $105(52.0)$ & \\
\hline
\end{tabular}

$B M I$ body mass index. The following factors are related to breast cancer incidence and were measured by the questionnaire: age, race, BMI, educational level, marital status, religion, family tumor history, and occupation. The two groups were comparable on these factors, although no significant between-group differences were found

\section{Characteristics of food preferences based on CM theory}

Tables 2 and 3 showed that there was a significant between-group difference in the frequency distribution of the preferences for the three food types $(P<0.001)$. We analyzed the between-group differences in food preferences of hot, neutral, and cold using logistic regression with age as a variable. The consumption of hot foods such as roast meat, shrimp, shellfish, soft drinks, popcorn/fries, and candies was significantly different between groups. Participants with a strong preference for roast meat, shrimp, shellfish, and popcorn/fries had 6-, 9.2-, 13.3-, and 7.6-times higher risk, respectively, of having breast cancer compared with participants with an average preference for these foods. Women who preferred soft drinks had a 4.9-times higher risk of having breast cancer compared with women who had an average
Table 2 Frequency distribution of preference for foods with hot, neutral and cold characteristics ( $N=404, \%)$

\begin{tabular}{lllll}
\hline Groups & \multicolumn{2}{l}{ Food nature } & & \\
\cline { 2 - 4 } & Hot (warm) & Neutral & Cold (cool) & \\
\hline Controls & $14(6.9)$ & $121(59.9)$ & $67(33.2)$ & $<0.001^{*}$ \\
Cases & $53(26.4)$ & $103(50.8)$ & $46(22.8)$ & \\
\hline
\end{tabular}

The $P$ value was $<0.05$, indicating a statistically significant between-group difference. The results showed a possible association between consumption of hot foods and a higher risk of breast cancer

* $P$ value was calculated by Chi square test

preference. Higher preferences for nuts and freshwater fish (neutral) and tofu and squid (cold) showed a lower OR for breast cancer (Table 4). 
Table 3 Specific frequency distribution of preference for foods with hot, neutral and cold characteristics $(N=404, \%)$

\begin{tabular}{|c|c|c|c|c|c|c|c|c|}
\hline Food nature & Food & $\begin{array}{l}\text { Breast } \\
\text { cancer }\end{array}$ & $\begin{array}{l}\text { Very } \\
\text { preferred }\end{array}$ & Preferred & Average & $\begin{array}{l}\text { Not } \\
\text { preferred }\end{array}$ & $\begin{array}{l}\text { Not very } \\
\text { preferred }\end{array}$ & $\begin{array}{l}\text { Statistical } \\
\text { significance }\end{array}$ \\
\hline \multirow[t]{38}{*}{ Hot } & \multirow[t]{2}{*}{ Spicy food } & No & $14(6.9)$ & $50(24.8)$ & $82(40.6)$ & $43(21.3)$ & $13(6.4)$ & 0.655 \\
\hline & & Yes & $19(9.4)$ & $55(27.2)$ & $68(33.7)$ & $46(22.8)$ & $14(6.9)$ & \\
\hline & \multirow[t]{2}{*}{ Roast meat } & No & $5(2.5)$ & $59(29.2)$ & $113(55.9)$ & $22(10.9)$ & $3(1.5)$ & 0.237 \\
\hline & & Yes & $12(5.9)$ & 68 (33.7) & $104(51.5)$ & $17(8.4)$ & $1(0.5)$ & \\
\hline & \multirow[t]{2}{*}{ Beef } & No & $16(8.0)$ & $58(28.9)$ & $83(41.3)$ & $29(14.4)$ & $15(7.5)$ & 0.534 \\
\hline & & Yes & $24(12.1)$ & 48 (23.6) & $87(43.2)$ & $31(15.1)$ & $12(6.0)$ & \\
\hline & \multirow[t]{2}{*}{ Mutton } & No & $8(4.0)$ & $32(16.0)$ & $83(41.0)$ & $50(24.5)$ & $29(14.5)$ & 0.777 \\
\hline & & Yes & $11(5.6)$ & $33(16.3)$ & $72(35.7)$ & $51(25.0)$ & 35 (17.3) & \\
\hline & \multirow[t]{2}{*}{ Chicken } & No & $36(17.8)$ & $102(50.5)$ & $55(27.2)$ & $8(4.0)$ & $1(0.5)$ & 0.138 \\
\hline & & Yes & $56(27.8)$ & $82(40.4)$ & $54(26.8)$ & $8(4.0)$ & $2(1.0)$ & \\
\hline & \multirow[t]{2}{*}{ Salmon/tuna } & No & $30(14.9)$ & $87(43.3)$ & $57(28.4)$ & $19(9.5)$ & $7(3.5)$ & 0.687 \\
\hline & & Yes & $37(18.2)$ & $76(37.4)$ & 65 (32.3) & $18(9.1)$ & $6(3.0)$ & \\
\hline & \multirow[t]{2}{*}{ Shrimp } & No & $13(6.5)$ & $53(26.4)$ & $84(41.3)$ & 38 (18.9) & $14(7.0)$ & $<0.001 *$ \\
\hline & & Yes & $44(21.9)$ & 60 (29.9) & $73(6.3)$ & $19(9.5)$ & $5(2.5)$ & \\
\hline & \multirow[t]{2}{*}{ Shellfish } & No & $9(4.5)$ & $37(18.3)$ & $97(48.0)$ & 48 (23.8) & $11(5.4)$ & $<0.001 *$ \\
\hline & & Yes & $31(15.2)$ & $56(27.8)$ & $73(36.4)$ & 39 (19.2) & $3(1.5)$ & \\
\hline & \multirow[t]{2}{*}{ Dark Chinese tea } & No & $30(14.9)$ & 76 (37.6) & $73(36.1)$ & $15(7.4)$ & $8(4.0)$ & 0.071 \\
\hline & & Yes & $31(15.2)$ & $73(36.0)$ & $67(33.0)$ & $30(14.7)$ & $2(1.0)$ & \\
\hline & \multirow{2}{*}{$\begin{array}{l}\text { Coffee/tea with } \\
\text { milk }\end{array}$} & No & $38(18.8)$ & $52(25.7)$ & $52(25.7)$ & $44(21.8)$ & $16(7.9)$ & 0.678 \\
\hline & & Yes & $43(21.5)$ & $54(26.5)$ & $56(27.5)$ & $32(16.0)$ & $17(8.5)$ & \\
\hline & \multirow[t]{2}{*}{ Soft drinks } & No & $3(1.5)$ & $10(5.0)$ & $65(32.2)$ & $92(45.5)$ & $31(15.3)$ & $0.001 *$ \\
\hline & & Yes & $10(5.0)$ & $30(14.9)$ & $72(35.8)$ & $65(32.3)$ & 24 (11.9) & \\
\hline & \multirow[t]{2}{*}{ Milk } & No & $10(5.0)$ & $49(24.4)$ & $91(44.8)$ & $35(17.4)$ & $17(8.5)$ & $0.014^{*}$ \\
\hline & & Yes & $25(12.2)$ & $37(18.3)$ & $90(44.7)$ & $43(21.3)$ & $7(3.6)$ & \\
\hline & \multirow[t]{2}{*}{ Alcohol } & No & $0(0.0)$ & $4(2.0)$ & $21(10.4)$ & $58(28.7)$ & $117(57.9)$ & 0.257 \\
\hline & & Yes & $3(1.5)$ & $2(1.0)$ & $17(8.5)$ & $66(32.5)$ & $114(56.5)$ & \\
\hline & \multirow[t]{2}{*}{ Beer } & No & $2(1.0)$ & $23(11.4)$ & $64(31.7)$ & $48(23.8)$ & $64(31.7)$ & 0.075 \\
\hline & & Yes & $6(3.0)$ & $15(7.5)$ & $47(23.1)$ & 65 (32.2) & $69(34.2)$ & \\
\hline & \multirow[t]{2}{*}{ Popcorn/fries } & No & $3(1.5)$ & 34 (16.8) & 79 (39.1) & $57(28.2)$ & $29(14.4)$ & $0.035^{*}$ \\
\hline & & Yes & $15(7.6)$ & $32(15.7)$ & $86(42.4)$ & $46(22.7)$ & $23(11.6)$ & \\
\hline & \multirow[t]{2}{*}{ Chocolate } & No & $25(12.4)$ & $61(30.3)$ & $82(40.8)$ & $26(12.9)$ & $7(3.5)$ & 0.842 \\
\hline & & Yes & $25(12.4)$ & $64(31.8)$ & $72(35.8)$ & $31(15.4)$ & $9(4.5)$ & \\
\hline & \multirow[t]{2}{*}{ Candies } & No & $3(1.5)$ & $21(10.4)$ & $93(46.0)$ & $67(33.2)$ & $18(8.9)$ & 0.250 \\
\hline & & Yes & $7(3.5)$ & $33(16.4)$ & 80 (39.8) & $65(32.3)$ & $16(8.0)$ & \\
\hline & \multirow[t]{2}{*}{ Deep boiled soup } & No & $59(29.2)$ & $98(48.5)$ & $38(18.8)$ & $5(2.5)$ & $2(1.0)$ & 0.759 \\
\hline & & Yes & $56(27.9)$ & $104(51.7)$ & 38 (18.9) & $2(1.0)$ & $1(0.5)$ & \\
\hline & \multirow[t]{2}{*}{ Western soup } & No & $13(6.5)$ & $73(36.3)$ & $81(40.3)$ & $22(10.9)$ & $12(6.0)$ & 0.059 \\
\hline & & Yes & $18(9.0)$ & $51(25.4)$ & $86(42.8)$ & $37(18.4)$ & $9(4.5)$ & \\
\hline Neutral & Fruits and vegeta- & No & 44 (21.6) & $131(64.8)$ & $25(12.6)$ & $2(1.0)$ & $0(0.0)$ & 0.452 \\
\hline & bles & Yes & $41(20.2)$ & $121(60.1)$ & $37(18.1)$ & $3(1.6)$ & $0(0.0)$ & \\
\hline & Pork & No & $15(7.5)$ & $95(46.8)$ & $83(41.3)$ & $5(2.5)$ & $4(2.0)$ & 0.690 \\
\hline & & Yes & $18(9.0)$ & $94(46.5)$ & $86(42.5)$ & $2(1.0)$ & $2(1.0)$ & \\
\hline & Pork with fat & No & $4(2.0)$ & $17(8.4)$ & $70(34.7)$ & $66(32.7)$ & $45(22.3)$ & 0.121 \\
\hline & & Yes & $4(2.0)$ & $32(16.0)$ & $72(35.5)$ & $63(31.0)$ & $31(15.5)$ & \\
\hline & Geese/duck & No & $5(2.5)$ & $45(22.3)$ & $101(50.0)$ & $36(17.8)$ & $15(7.4)$ & 0.209 \\
\hline & & Yes & $13(6.5)$ & $55(27.0)$ & $92(45.5)$ & $31(15.5)$ & $11(5.5)$ & \\
\hline & Fresh water fish & No & $19(9.4)$ & 79 (39.1) & 80 (39.6) & $16(7.9)$ & $7(3.5)$ & 0.140 \\
\hline & & Yes & $10(5.0)$ & $63(31.3)$ & $100(49.3)$ & $20(10.0)$ & $9(4.5)$ & \\
\hline
\end{tabular}


Table 3 continued

\begin{tabular}{|c|c|c|c|c|c|c|c|c|}
\hline Food nature & Food & $\begin{array}{l}\text { Breast } \\
\text { cancer }\end{array}$ & $\begin{array}{l}\text { Very } \\
\text { preferred }\end{array}$ & Preferred & Average & $\begin{array}{l}\text { Not } \\
\text { preferred }\end{array}$ & $\begin{array}{l}\text { Not very } \\
\text { preferred }\end{array}$ & $\begin{array}{l}\text { Statistical } \\
\text { significance }\end{array}$ \\
\hline & Nuts & No & $39(19.5)$ & $84(41.5)$ & $64(31.5)$ & $10(5.0)$ & $5(2.5)$ & $0.020^{*}$ \\
\hline & & Yes & $26(12.6)$ & $65(32.2)$ & $88(43.7)$ & $18(9.0)$ & $5(2.5)$ & \\
\hline & Boiling soup & No & $37(18.3)$ & $98(48.5)$ & $57(28.2)$ & $6(3.0)$ & $4(2.0)$ & 0.233 \\
\hline & & Yes & $31(15.5)$ & $90(44.5)$ & $61(30.0)$ & $16(8.0)$ & $4(2.0)$ & \\
\hline \multirow[t]{12}{*}{ Cold } & \multirow{2}{*}{$\begin{array}{l}\text { Fruits and vegeta- } \\
\text { bles }\end{array}$} & No & 38 (19.0) & $117(58.0)$ & $45(22.5)$ & $1(0.5)$ & $0(0.0)$ & 0.323 \\
\hline & & Yes & $30(14.9)$ & $11(56.9)$ & $53(26.1)$ & $4(2.1)$ & $0(0.0)$ & \\
\hline & \multirow[t]{2}{*}{ Sea fish } & No & 35 (17.3) & $97(48.0)$ & $58(28.7)$ & $10(5.0)$ & $2(1.0)$ & 0.963 \\
\hline & & Yes & $31(15.5)$ & $102(50.5)$ & $57(28.0)$ & $9(4.5)$ & $3(1.5)$ & \\
\hline & \multirow[t]{2}{*}{ Squid } & No & $27(13.6)$ & $59(29.1)$ & $85(42.2)$ & 25 (12.6) & $5(2.5)$ & 0.219 \\
\hline & & Yes & $19(9.5)$ & $45(22.1)$ & $96(47.7)$ & $36(17.6)$ & $6(3.0)$ & \\
\hline & \multirow{2}{*}{$\begin{array}{l}\text { Products made } \\
\text { from bean }\end{array}$} & No & $53(26.4)$ & $103(50.7)$ & $39(19.4)$ & $5(2.5)$ & $2(1.0)$ & $0.019 *$ \\
\hline & & Yes & $39(19.1)$ & $87(43.2)$ & $67(33.2)$ & $8(4.0)$ & $1(0.5)$ & \\
\hline & \multirow[t]{2}{*}{ Light Chinese tea } & No & $28(13.9)$ & $82(40.6)$ & $64(31.7)$ & $23(11.4)$ & $5(2.5)$ & 0.148 \\
\hline & & Yes & $33(16.1)$ & $64(31.7)$ & 78 (38.7) & $26(13.1)$ & $1(0.5)$ & \\
\hline & \multirow[t]{2}{*}{ Herbal tea } & No & $4(2.0)$ & $40(19.8)$ & $111(55.0)$ & 39 (19.3) & $8(4.0)$ & 0.582 \\
\hline & & Yes & $5(2.5)$ & $36(17.7)$ & $101(50.0)$ & $47(23.2)$ & $13(6.6)$ & \\
\hline
\end{tabular}

The $P$ value was $<0.05$, indicating a statistically significant between-group difference. The results showed a possible association between consumption of hot foods and a higher risk of breast cancer

* P values were calculated by Chi square tests

\section{Association between CM dietary patterns or habits and risk of breast cancer}

A higher frequency of eating out for breakfast (4-5 times per week, $P=0.015$; $6-7$ times per week, $P<0.001)$ and lunch (4-5 times per week, $P<0.001 ; 6-7$ times per week, $P=0.006$ ) was positively associated with the risk of breast cancer. In general, participants who dined out for breakfast and lunch (at least four times per week) had at least twice the risk of having breast cancer compared with participants who dined out 0-1 time per week (Table 5). A higher frequency of Guangdong dim sum/tea drinking was positively associated with a higher risk of breast cancer. Participants who ate dim sum more than three times per week were 2.8 times higher in frequency to be at risk of breast cancer than those who ate it less than once a week (1-2 times per week, $P=0.05$; $>3$ times per week, $P<0.001$ ) (Table 6).

\section{Consumption of CM supplements, Chinese soup, and risk of breast cancer}

The results indicated significant positive associations of the consumption of CM supplements and Chinese soup with a lower risk of breast cancer (Table 7).

\section{Discussion}

CM theory offers a way of classifying food based on its properties. When the balance of Yin and Yang in the body is upset, many diseases can develop, including breast cancer $[14,16]$. Although different from modern nutritional classification theories, CM-based food classification could help people to improve their dietary patterns by eating more neutral or cold pattern foods and reducing consumption of high-risk hot foods to prevent breast cancer. Different food characteristics can alter the balance of Yin and Yang in the body. Numerous experimental studies have demonstrated that some food based on CM classifications may reduce the risk of cancer [8, 16-20]. However, no epidemiological research has reported a relationship between CM-defined dietary patterns and breast cancer morbidity.

In this study, participants with breast cancer shared a preference for a hot-patterned diet containing fried foods and meat rather than vegetables. This may have contributed to their breast cancer. The participants with breast cancer also had a relatively higher frequency of dining out, eating fast food, and consuming soft drinks, coffee, milk, and other foods classified as hot according to CM theory. In CM, hot food is defined as food that is high in energy and low in fiber, such as beef and prawns, or food that is fried or roasted $[8,21]$. This is consistent with the etiology and pathogenesis of breast cancer in Western medicine [22]. It was demonstrated that carcinogenic substances found in foods according to Western medicine mostly belong to foods classified as hot according to CM, such as barbecued foods, red meat, fatty meat, and prawns [23-27]. 
Table 4 Odds ratio and its corresponding $95 \%$ confidence intervals of having breast cancer by eating different food

\begin{tabular}{|c|c|c|c|c|c|}
\hline & Controls N = 202 & Cases $\mathrm{N}=202$ & OR & $95 \% \mathrm{Cl}$ & $P$ value \\
\hline \multicolumn{6}{|l|}{ Shrimp } \\
\hline Average & 84 & 73 & 1.00 & Reference & \\
\hline Much preferred & 13 & 44 & 3.90 & $1.95-7.79$ & $<0.001 *$ \\
\hline Preferred & 53 & 60 & 1.30 & $0.80-2.12$ & 0.285 \\
\hline Not preferred & 38 & 19 & 0.58 & $0.31-1.08$ & 0.086 \\
\hline Not preferred much & 14 & 5 & 0.41 & $0.14-1.20$ & 0.094 \\
\hline \multicolumn{6}{|l|}{ Shellfish } \\
\hline Average & 97 & 73 & 1.00 & Reference & \\
\hline Much preferred & 9 & 31 & 4.58 & $2.05-10.21$ & $<0.001^{*}$ \\
\hline Preferred & 37 & 56 & 2.01 & $1.20-3.36$ & $0.007^{*}$ \\
\hline Not preferred & 48 & 39 & 1.08 & $0.64-1.82$ & 0.773 \\
\hline Not preferred much & 11 & 3 & 0.36 & $0.10-1.35$ & 0.116 \\
\hline \multicolumn{6}{|l|}{ Soft drinks } \\
\hline Average & 65 & 72 & 1.00 & Reference & \\
\hline Much preferred & 3 & 10 & 3.01 & $0.79-11.41$ & 0.092 \\
\hline Preferred & 10 & 30 & 2.71 & $1.23-5.97$ & $0.011^{*}$ \\
\hline Not preferred & 92 & 65 & 0.64 & $0.40-1.01$ & 0.056 \\
\hline Not preferred much & 31 & 24 & 0.70 & $0.37-1.31$ & 0.264 \\
\hline \multicolumn{6}{|l|}{ Milk } \\
\hline Average & 91 & 90 & 1.00 & Reference & \\
\hline Much preferred & 10 & 25 & 2.53 & $1.15-5.57$ & $0.018^{*}$ \\
\hline Preferred & 49 & 37 & 0.76 & $0.46-1.28$ & 0.306 \\
\hline Not preferred & 35 & 43 & 1.24 & $0.73-2.12$ & 0.425 \\
\hline Not preferred much & 17 & 7 & 0.42 & $0.17-1.05$ & 0.058 \\
\hline \multicolumn{6}{|l|}{ Popcorn/fries } \\
\hline Average & 79 & 86 & 1.00 & Reference & \\
\hline Much preferred & 3 & 15 & 4.59 & $1.28-16.46$ & $0.011^{*}$ \\
\hline Preferred & 34 & 32 & 0.87 & $0.49-1.53$ & 0.618 \\
\hline Not preferred & 57 & 46 & 0.74 & $0.45-1.22$ & 0.235 \\
\hline Not preferred much & 29 & 23 & 0.73 & $0.39-1.36$ & 0.321 \\
\hline \multicolumn{6}{|l|}{ Nuts } \\
\hline Average & 64 & 88 & 1.00 & Reference & \\
\hline Much preferred & 39 & 26 & 0.49 & $0.27-0.88$ & $0.016^{*}$ \\
\hline Preferred & 84 & 65 & 0.56 & $0.36-0.89$ & $0.013^{*}$ \\
\hline Not preferred & 10 & 18 & 1.31 & $0.57-3.02$ & 0.528 \\
\hline Not preferred much & 5 & 5 & 0.73 & $0.20-2.62$ & 0.745 \\
\hline \multicolumn{6}{|c|}{ Products made from bean } \\
\hline Average & 39 & 67 & 1.00 & Reference & \\
\hline Much preferred & 53 & 39 & 0.43 & $0.24-0.76$ & $0.003^{*}$ \\
\hline Preferred & 103 & 87 & 0.49 & $0.30-0.80$ & $0.004^{*}$ \\
\hline Not preferred & 5 & 8 & 0.93 & $0.29-3.05$ & 1.000 \\
\hline Not preferred much & 2 & 1 & 0.29 & $0.026-3.32$ & 0.555 \\
\hline
\end{tabular}

A statistically significant $\mathrm{OR}$ (odds ratio) $>1$ indicates a risk factor; an $\mathrm{OR}<1$ indicates a prevention factor

* indicates a statistically significant between-group diference

Depending on the seasons and individual constitution, people in Hong Kong will choose different foods, herbs, or soups to adjust the functions of the organs and balance the Yin and Yang of the body [28, 29]. Our findings suggested that frequent consumption of Chinese herbs or Chinese soup is inversely associated with the risk of breast cancer. CM dietary herbs (supplements) or Chinese herbal medicine may play a crucial role in 
Table 5 Adjusted odds ratio and its corresponding 95\% confidence intervals of having breast cancer by dining out patterns (fast food)

\begin{tabular}{|c|c|c|c|c|c|}
\hline & Controls $n=202$ & Cases $n=202$ & Adjusted OR & $95 \% \mathrm{Cl}$ & $P$ value \\
\hline \multicolumn{6}{|l|}{ Breakfast } \\
\hline 0-1 times per week & 113 & 81 & 1.00 & Reference & \\
\hline 2-3 times per week & 39 & 34 & 1.22 & $0.71-2.09$ & 0.479 \\
\hline 4-5 times per week & 19 & 30 & 2.20 & $1.16-4.18$ & $0.015^{*}$ \\
\hline 6-7 times per week & 31 & 56 & 2.52 & $1.49-4.25$ & $<0.001^{*}$ \\
\hline \multicolumn{6}{|l|}{ Lunch } \\
\hline 0-1 times per week & 68 & 40 & 1.00 & Reference & \\
\hline 2-3 times per week & 56 & 59 & 1.70 & $0.99-2.91$ & 0.053 \\
\hline 4-5 times per week & 31 & 44 & 3.24 & $1.80-5.80$ & $<0.001^{*}$ \\
\hline 6-7 times per week & 47 & 59 & 2.13 & $1.24-3.69$ & $0.006^{*}$ \\
\hline
\end{tabular}

Adjusted for age and body mass index (BMI)

* The $P$ value was $<0.05$, indicating a statistically significant between-group difference

Table 6 Adjusted odds ratio and its corresponding $95 \%$ confidence intervals of having breast cancer by frequency of Guangdong drinking tea

\begin{tabular}{|c|c|c|c|c|c|}
\hline & Controls $n=202$ & Cases $n=202$ & Adjusted OR & $95 \% \mathrm{Cl}$ & $P$ value \\
\hline \multicolumn{6}{|l|}{ Guangdong tea (Yum Cha) } \\
\hline Less than once per week & 142 & 109 & 1.00 & Reference & \\
\hline $1-2$ times per week & 46 & 56 & 1.59 & $1.00-2.52$ & $0.05^{*}$ \\
\hline$>3$ times per week & 12 & 35 & 3.80 & $1.88-7.66$ & $<0.001^{*}$ \\
\hline
\end{tabular}

The $P$ value was $<0.05$, indicating a statistically significant between-group difference

* Adjusted for age and body mass index (BMI)

Table 7 Adjusted odds ratio and its corresponding $95 \%$ confidence intervals of having breast cancer by the dietary patterns of Chinese medicine and related supplement and soup intake

\begin{tabular}{|c|c|c|c|c|c|}
\hline & $\begin{array}{l}\text { Controls } \\
n=202\end{array}$ & $\begin{array}{l}\text { Cases } \\
n=202\end{array}$ & $\begin{array}{l}\text { Adjusted } \\
\text { OR }\end{array}$ & $95 \% \mathrm{Cl}$ & $P$ value \\
\hline \multicolumn{6}{|c|}{ Chinese medicine } \\
\hline Seldom & 98 & 112 & 1.00 & Reference & \\
\hline $\begin{array}{l}\text { Some- } \\
\text { times }\end{array}$ & 61 & 51 & 0.73 & $0.46-1.16$ & 0.183 \\
\hline Often & 43 & 36 & 0.73 & $0.44-1.23$ & 0.240 \\
\hline \multicolumn{6}{|c|}{ Food supplement } \\
\hline Seldom & 119 & 140 & 1.00 & Reference & \\
\hline $\begin{array}{l}\text { Some- } \\
\text { times }\end{array}$ & 35 & 32 & 0.78 & $0.45-1.33$ & 0.358 \\
\hline Often & 47 & 26 & 0.47 & $0.28-0.81$ & $0.005^{*}$ \\
\hline \multicolumn{6}{|c|}{ Chinese soup } \\
\hline Seldom & 91 & 115 & 1.00 & Reference & \\
\hline $\begin{array}{l}\text { Some- } \\
\text { times }\end{array}$ & 83 & 69 & 0.66 & $0.43-1.00$ & 0.051 \\
\hline Often & 28 & 16 & 0.45 & $0.23-0.89$ & $0.019^{*}$ \\
\hline
\end{tabular}

The $P$ value was $<0.05$, indicating a statistically significant between-group difference

* Adjusted for age and body mass index (BMI) preventing the incidence, recurrence, and metastasis of breast cancer. Many experimental studies have demonstrated that some foods representative of the CM classifications could reduce the risk of cancer [30-32], and that there is a relationship between the use of $\mathrm{CM}$ herbs or related Chinese herbal medicine and the reduced incidence of breast cancer [33].

The design and conduct of this study had some limitations. This study did not examine other factors related to breast cancer, such as whether or not participants exercised regularly or whether they used oral contraceptives. In addition, because the control group was recruited from health workshops, and included both healthy and unhealthy women, there may be a health worker effect resulting in selection bias. Because of limited resources, we recruited only 37 people to our pilot study. This sample size was not large enough to conduct a factor analysis to assess the internal consistency of the questionnaire. Because this questionnaire was the first to analyze dietary patterns in terms of hot, neutral, and cold characteristics as defined by $\mathrm{CM}$, there was no other standard questionnaire and results for comparison. The evidence strength in terms of epidemiology is also insufficient. 


\section{Conclusions}

Non-breast cancer participants adopted a healthy and balanced (neutral) dietary pattern, along with consumption of CM supplements and Guangdong soups.

\section{Additional files}

Additional file 1. Classification and characteristics of foods according to TCM theory.

Additional file 2. Reference food questionnaire.

Additional file 3. Minimum standards of reporting checklist.

Additional file 4. Questionnaire-N-dietary.

Additional file 5. IRB.

\section{Abbreviations}

CM: Chinese medicine; FFQ: Food Frequency Questionnaires; CSCMHKU: Clinic Center of School of Chinese Medicine, The University of Hong Kong; BMI: body mass index.

\section{Authors' contributions}

JPC conceived and designed the study. XZ, JSY, JY, FZM and KCT recruited the participants. WTYL revised the questionnaire. XZ and TX collected the data. $Z Y X, L X L$ and JSY performed the data analysis. FG performed the literature search. JPC and XZ wrote the manuscript. TX, ZYX, JSY, JY, KCT and FG revised the manuscript. All authors read and approved the final manuscript.

\section{Author details}

1 School of Chinese Medicine, The University of Hong Kong, Hong Kong, China. ${ }^{2}$ Department of Dermatology, Guangzhou University of Chinese Medicine, Guangzhou 510020, China. ${ }^{3}$ School of Public Health, Peking University, Beijing, China. ${ }^{4}$ The Hong Kong Associate of Chinese Medicine, Hong Kong, China. ${ }^{5}$ Chengdu University of Traditional Chinese Medicine, Chengdu 510020, China.

\section{Acknowledgements}

Thanks a lot of Prof. Y Tong for the application IRB, Ms. Xue Huitian and Ms. Wong Mei Kuen for the provision of statistics. We are grateful to all the study participants for their contributions and support. Incident breast cancer cases for this study were collected by the School of Chinese Medicine, the University of Hong Kong.

\section{Competing interests}

The authors declare that they have no competing interests.

\section{Availability of data and materials}

Please contact author for data requests.

\section{Ethics approval and consent to participate}

Prior to commencement of the study, ethical approval (UW 05-211T/874) (Additional file 5) was obtained from the Research Ethics Board of the University of Hong Kong.

\section{Publisher's Note}

Springer Nature remains neutral with regard to jurisdictional claims in published maps and institutional affiliations.

Received: 11 April 2017 Accepted: 6 June 2017

Published online: 29 June 2017

\section{References}

1. Hospital Authority: Hong Kong Cancer Registry web site. http://www3. ha.org.hk/cancereg/statistics.html.
2. Wang $L$, Wang J, Wang $M$, et al. Using Internet search engines to obtain medical information: a comparative study. J Med Internet Res. 2012;14:e74.

3. Sasco AJ. Breast cancer and the environment. Horm Res. 2003;60(Suppl 3):50.

4. Li C, Zhao X, Toline EC, Siegal GP, Evans LM, Ibrahim-Hashim A, Desmond RA, Hardy RW. Prevention of carcinogenesis and inhibition of breast cancer tumor burden by dietary stearate. Carcinogenesis. 2011;4:1-8.

5. Evans LM, Toline EC, Desmond R, Siegal GP, Hashim Al, Hardy RW. Dietary stearate reduces human breast cancer metastasis burden in athymic nude mice. Clin Exp Metastasis. 2009;26:415-24.

6. Leung J. The dieting phenomenon in Hong Kong: the changing attitudes towards dieting among young women in Hong Kong. In: Civic exchange. 6; 2002.

7. Crozier SR, Robinson SM, Godfrey KM, Cooper C, Inskip HM. Women's dietary patterns change little from before to during pregnancy. J Nutr. 2009;139:1956-63.

8. Lee MM, Shen JM. Dietary patterns using traditional Chinese medicine principles in epidemiological studies. Asia Pac J Clin Nutr. 2008;17(Suppl 1):79-81.

9. Pierce JP. Diet and breast cancer prognosis: making sense of the women's healthy eating and living and women's intervention nutrition study trials. Curr Opin Obstet Gyn. 2009;21(1):86-91.

10. Martinez-Chacin RC, Keniry M, Dearth RK. Analysis of high fat diet induced genes during mammary gland development: identifying role players in poor prognosis of breast cancer. BMC Res Notes. 2014;18(7):543.

11. De Lorgeril M, Salen P. Do statins increase and Mediterranean diet decrease the risk of breast cancer? BMC Med. 2014;5(12):94

12. Hu FB, Rimm E, Smith-Warner SA. Reproducibility and validity of dietary patterns assessed with a food-frequency questionnaire. Am J Clin Nutr. 1999;69(2):243-9.

13. Kroenke Candyce H, Fung Teresa T, Hu Frank B, Holmes Michelle D. Dietary patterns and survival after breast cancer diagnosis. J Clin Oncol. 2005;23(36):9295-303.

14. Zhang CX, Ho SC, Fu JH, Cheng SZ, Chen YM, Lin FY. Dietary patterns and breast cancer risk among Chinese women. Cancer Causes Control. 2011;22(1):115-24.

15. Lv N, Brown JL. Chinese American family food systems: impact of Western influences. J Nutr Educ Behav. 2010;42(2):106-14.

16. Miller PE, Morey MC, Hartman TJ, Snyder DC, Sloane R, Cohen HJ, DemarkWahnefried W. Dietary patterns differ between urban and rural older, long-term survivors of breast, prostate, and colorectal cancer and are associated with body mass index. J Acad Nutr Diet. 2012;112(6):824-31.

17. Pierce JP, Faerber S, Wright FA, Newman V, Flatt SW, Kealey S, Rock $\mathrm{CL}$, Hryniuk W, Greenberg ER. Feasibility of a randomized trial of a high-vegetable diet to prevent breast cancer recurrence. Nutr Cancer. 1997;28(3):282-8.

18. Moran LJ, Hutchison SK, Norman RJ, Teede HJ. Lifestyle changes in women with polycystic ovary syndrome. Cochrane Database Syst Rev. 2011;16(2):CD007506.

19. Brunner EJ, Rees K, Ward K, Burke M, Thorogood M. Dietary advice for reducing cardiovascular risk. Cochrane Database Syst Rev. 2007;17(4):CD002128.

20. Schatzkin A. Dietary change as a strategy for preventing cancer. Cancer Metastasis Rev. 1997:16(3-4):377-92.

21. Huang LP, Zhu MF, Yu RY, Du JQ, Liu HN. Study on discrimination mode of cold and hot properties of traditional Chinese medicines based on biological effects. China J Chin Mater Med. 2014;39(17):3353-8.

22. Reuss-Borst M, Kötter J, Hartmann U, Füger-Helmerking G, Weiß J. Nutrition patterns in German breast cancer patients. Dtsch Med Wochenschr. 2011;136(12):575-81.

23. Zhang CX, Ho SC, Fu JH, Cheng SZ, Chen YM, Lin FY. Dietary patterns and breast cancer risk among Chinese women. Cancer Cause Control. 2011;22(1):115-24.

24. Cui X, Dai Q, Tseng M, Shu XO, Gao YT, Zheng W. Dietary patterns and breast cancer risk in the shanghai breast cancer study. Cancer Epidemiol Biomark. 2007; 16:1443-8.

25. Zhang CX, Ho SC, Chen YM, Lin FY, Fu JH, Cheng SZ. Meat and egg consumption and risk of breast cancer among Chinese women. Cancer Cause Control. 2009;20:1845-53. 
26. Andrici J, Eslick GD. Hot food and beverage consumption and the risk of esophageal cancer: a meta-analysis. Am J Prev Med. 2015;49(6):952-60.

27. Turner LB. A meta-analysis of fat intake, reproduction, and breast cancer risk: an evolutionary perspective. Am J Hum Biol. 2011;23(5):601-8.

28. Ou B, Huang D, Hampsch-Woodill M, Flanagan JA. When east meets west: the relationship between yin-yang and antioxidation-oxidation. Faseb J. 2003;17(2):127-9.

29. Wang J, Tang YL. On the concept of health in traditional Chinese medicine and its characteristics and advantages. Chin J Med Hist. 2011;40(1):13-4.
30. Fornier MN. Approved agents for metastatic breast cancer. Semin Oncol. 2011;38(Suppl 2):S3-10.

31. Cuzick J, DeCensi A, Arun B, Brown PH, Castiglione M, Dunn B, Forbes JF, Glaus A, Howell A, von Minckwitz G, Vogel V, Zwierzina H. Preventive therapy for breast cancer: a consensus statement. Lancet Oncol. 2011;12(5):496-503.

32. Molokhia EA, Perkins A. Preventing cancer. Prim Care. 2008;35(4):609-23

33. Dayal HH, Kalia A. Preventing breast cancer in postmenopausal women by achievable diet modification: a missed opportunity in public health policy. Breast. 2010;19(5):309-11.

\section{Submit your next manuscript to BioMed Central and we will help you at every step:}

- We accept pre-submission inquiries

- Our selector tool helps you to find the most relevant journal

- We provide round the clock customer support

- Convenient online submission

- Thorough peer review

- Inclusion in PubMed and all major indexing services

- Maximum visibility for your research

Submit your manuscript at

www.biomedcentral.com/submit 